\title{
Correlation between electronic configuration and
}

magnetic stability in dysprosium single atom

\section{magnets}

Fabio Donati ${ }^{1,2, *}$, Marina Pivetta ${ }^{3}$, Christoph Wolf ${ }^{1,4}$, Aparajita Singha ${ }^{1,4,5}$, Christian

Wäckerlin ${ }^{3,6}$, Romana Baltic ${ }^{3}$, Edgar Fernandes ${ }^{3}$, Jean-Guillaume de Groot ${ }^{3}$, Safa Lamia

Ahmed $^{1,2}$, Luca Persichetti ${ }^{7,8}$, Corneliu Nistor $^{7}$, Jan Dreiser $^{9}$, Alessandro Barla $^{10}$, Pietro Gambardella ${ }^{7}$ Harald Brune ${ }^{3}$, and Stefano Rusponi ${ }^{3, *}$

${ }^{1}$ Center for Quantum Nanoscience, Institute for Basic Science (IBS), Seoul 03760, Republic of Korea

${ }^{2}$ Department of Physics, Ewha Womans University, Seoul 03760, Republic of Korea

${ }^{3}$ Institute of Physics, École Polytechnique Fédérale de Lausanne, Station 3, CH-1015 Lausanne, Switzerland

${ }^{4}$ Ewha Womans University, Seoul 03760, Republic of Korea

${ }^{5}$ Max Planck Institute for Solid State Research, Stuttgart, Germany

${ }^{6}$ Surface Science and Coating Technologies, Empa - Swiss Federal Laboratories for Materials Research and Technology, Überlandstrasse 129, 8600 Dübendorf, Switzerland 
${ }^{7}$ Department of Materials, ETH Zurich, Hönggerbergring 64, CH-8093 Zurich, Switzerland

${ }^{8}$ Department of Sciences, Roma Tre University, I-00146, Roma, Italy

${ }^{9}$ Swiss Light Source (SLS), Paul Scherrer Institute (PSI), CH-5232 Villigen PSI, Switzerland

${ }^{10}$ Istituto di Struttura della Materia (ISM), Consiglio Nazionale delle Ricerche (CNR), I-34149

Trieste, Italy

KEYWORDS: Single atom magnets, lanthanides, X-ray magnetic circular dichroism, scanning tunneling microscopy, density functional theory, multiplet calculations

\section{ABSTRACT}

Single atom magnets offer the possibility of magnetic information storage in the most fundamental unit of matter. Identifying the parameters that control the stability of their magnetic states is crucial to design novel quantum magnets with tailored properties. Here we use X-ray absorption spectroscopy to show that the electronic configuration of dysprosium atoms on $\mathrm{MgO}(100)$ thin films can be tuned by the proximity of the metal $\mathrm{Ag}(100)$ substrate onto which the $\mathrm{MgO}$ films are grown. Increasing the $\mathrm{MgO}$ thickness from 2.5 to 9 monolayers induces a change in the dysprosium electronic configuration from $4 f^{9}$ to $4 f^{10}$. Hysteresis loops indicate long magnetic lifetimes for both configurations, however, with a different field-dependent magnetic stability. Combining these measurements with scanning tunneling microscopy, density functional theory, and multiplet calculations unveils the role of the adsorption site and charge transfer to the substrate in determining the stability of quantum states in dysprosium single atom magnets. 


\section{MAIN TEXT}

Lanthanide based quantum magnets are model systems for atomic scale memory ${ }^{1-8}$ and quantum logic devices ${ }^{9,10}$. Due to their strong localization, their $4 \mathrm{f}$ electrons generally show very limited degree of covalency and preserve an atomic-like character with well-defined integer orbital occupation. Depending on the ligand environment, lanthanide complexes and surface-adsorbed atoms and can be mostly found in either atom-like $\left(4 \mathrm{f}^{\mathrm{n}}\right)^{4,11-15}$ or bulk-like configuration $\left(4 \mathrm{f}^{\mathrm{n}-1}\right)^{1-3 \text {, }}$ 16-23. Not only does the $4 \mathrm{f}$ occupation determine the magnetic moment and stability of the atom, but it also has an impact on the population of the $6 s^{2} \mathrm{~d}$ valence orbitals ${ }^{15,22,24,25}$ determining the behavior in spin transport measurements ${ }^{8,26-28}$. Hence, understanding how to control the lanthanide atom's electronic configuration is a crucial step to develop novel approaches to design quantum magnets with tailored stability and accessibility of their magnetic states ${ }^{22,29}$.

Atom adsorption on suitable surfaces is a viable pathway for creating such quantum magnets ${ }^{3,4}$, ${ }^{8}$ and allowing individual access to their spin states using transport techniques ${ }^{8,26-28,30}$. However, despite the relatively simple atom-support coordination structure, it remains challenging to theoretically predict how the substrate and adsorption geometry impact their multi-orbital spin configuration, calling for experiments to shed light on these mechanisms.

Among the lanthanide series, dysprosium (Dy) has been employed to realize molecular magnets with record-high blocking temperature ${ }^{1,2,19}$ and surface-supported single atom magnets with long spin lifetime $e^{4,8}$. Moreover, recent theoretical calculations on DyO complexes predict large magnetic anisotropy for Dy in both $4 \mathrm{f}^{9}$ and $4 \mathrm{f}^{10}$ electronic configurations $\mathrm{s}^{31,32}$. Here, we focus on O-coordinated Dy atoms resulting from low-temperature adsorption on $\mathrm{MgO}$ thin films grown on $\operatorname{Ag}(100)$. We show that the electronic configuration and, consequently, the magnetic properties of the Dy atoms depend on the thickness of the supporting $\mathrm{MgO}$ layer. For $\mathrm{MgO}$ layers with an 
average thickness $\mathrm{t}_{\mathrm{MgO}}<5$ monolayers (ML), X-ray absorption spectroscopy (XAS) and magnetic circular dichroism (XMCD) at $2.5 \mathrm{~K}$ reveal a predominance of bulk-like Dy $4 \mathrm{f}^{9}$ configuration, displaying magnetic hysteresis indicative of long lifetimes. By increasing the thickness of the $\mathrm{MgO}$ layer, Dy atoms with $4 \mathrm{f}^{10}$ configuration progressively increase in abundance and become the dominant species for $\mathrm{t}_{\mathrm{MgO}}>6$ 6-7 ML. Remarkably, Dy atoms show magnetic hysteresis also in this configuration, however, with a characteristic butterfly loop indicating quantum tunneling of the magnetization (QTM) in absence of an external field. Magnetic relaxation measurements reveal that the lifetime of magnetic states can be controlled using the external field, with a maximum of about $200 \mathrm{~s}$ for $0.25-0.40 \mathrm{~T}$. Density functional theory (DFT) and multiplet calculations reveal how charge transfer to the substrate and adsorption geometry determine the electronic configuration of Dy and the stability of its magnetic states.

We perform XAS/XMCD measurements at the $\mathrm{M}_{4,5}$ edges ( $3 \mathrm{~d} \rightarrow 4 \mathrm{f}$ transitions) of Dy at $6.8 \mathrm{~T}$ and $2.5 \mathrm{~K}$ at the EPFL/PSI X-Treme beamline at the Swiss Light Source ${ }^{33}$ to determine the $4 \mathrm{f}$ orbital occupation and magnetic properties. To avoid measurements on exposed $\operatorname{Ag}(100)$ regions, we use $\mathrm{MgO}$ films with $\mathrm{t}_{\mathrm{MgO}}>2 \mathrm{ML}$. XAS/XMCD spectra are acquired in two different geometries, i.e., with the photon beam and magnetic field oriented along the surface normal (normal incidence) and 60 degrees off-normal (grazing incidence, see SI for details), to determine the magnetization easy axis of the Dy atoms ${ }^{4,11,12,34}$.

The XAS and XMCD spectra as a function of the average $\mathrm{MgO}$ thickness are displayed in Figure 1a and $1 b$. The energy of the maximum at the $\mathrm{M}_{5}$ edge, both in XAS and XMCD, changes from $1290.5 \mathrm{eV}$ to $1288.3 \mathrm{eV}$ going from ultra-thin to thicker $\mathrm{MgO}$ layers. These two energies are characteristic of $4 \mathrm{f}^{9}$ and $4 \mathrm{f}^{10}$ electronic configurations, respectively, as deduced by comparison 
with previous data of Dy atoms on metals ${ }^{11}$ and supported graphene $e^{4,12}$. The continuous evolution implies the coexistence of $4 \mathrm{f}^{9}$ and $4 \mathrm{f}^{10}$ Dy atoms, whose proportions depend on the $\mathrm{MgO}$ thickness.
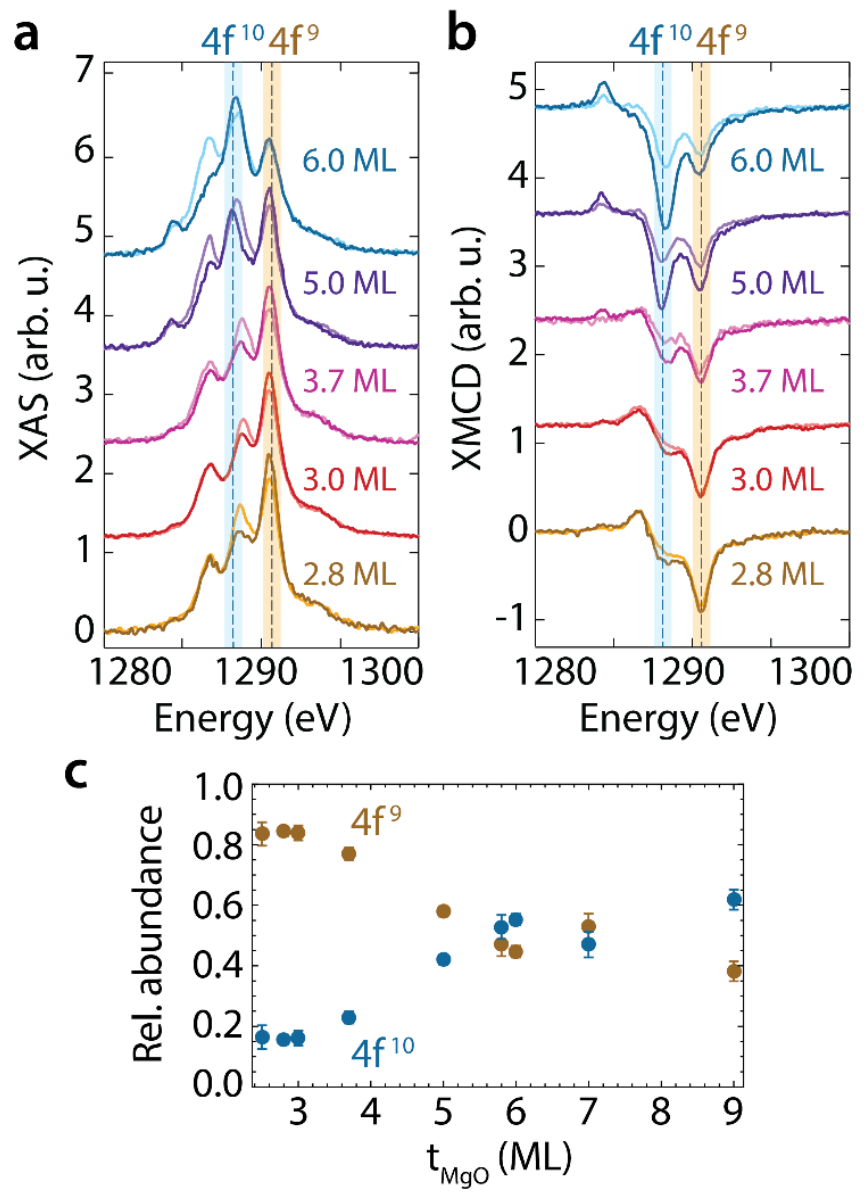

Figure 1. $4 \mathrm{f}$ occupation vs. $\mathrm{MgO}$ thickness for Dy atoms on $\mathrm{MgO} / \mathrm{Ag}(100)$. (a) $\mathrm{XAS}$ and (b) $\mathrm{XMCD}$ at the $\mathrm{Dy} \mathrm{M}_{5}$ edge as a function of the average $\mathrm{MgO}$ thickness $\mathrm{t}_{\mathrm{MgO}}$. Fractional values of $\mathrm{t}_{\mathrm{MgO}}$ indicate incomplete filling of the $\mathrm{MgO}$ atomic layers. Darker/lighter lines show the measurement at normal/grazing incidence, respectively. The position of the $4 \mathrm{f}^{9}$ and $4 \mathrm{f}^{10}$ main peaks of XAS and XMCD measurements are indicated with light blue and brown bands, respectively. (Dy coverage $\Theta_{\text {Dy }}=0.004 \div 0.014 \mathrm{ML}, \mathrm{B}=6.8 \mathrm{~T}, \mathrm{~T}=2.5 \mathrm{~K}$ ). (c) Relative abundance of the $4 \mathrm{f}^{9}$ and $4 \mathrm{f}^{10}$ species obtained by fitting the experimental data with simulated spectra from multiplet calculations (see SI for details). Standard deviation from the fit are displayed as error bars. 

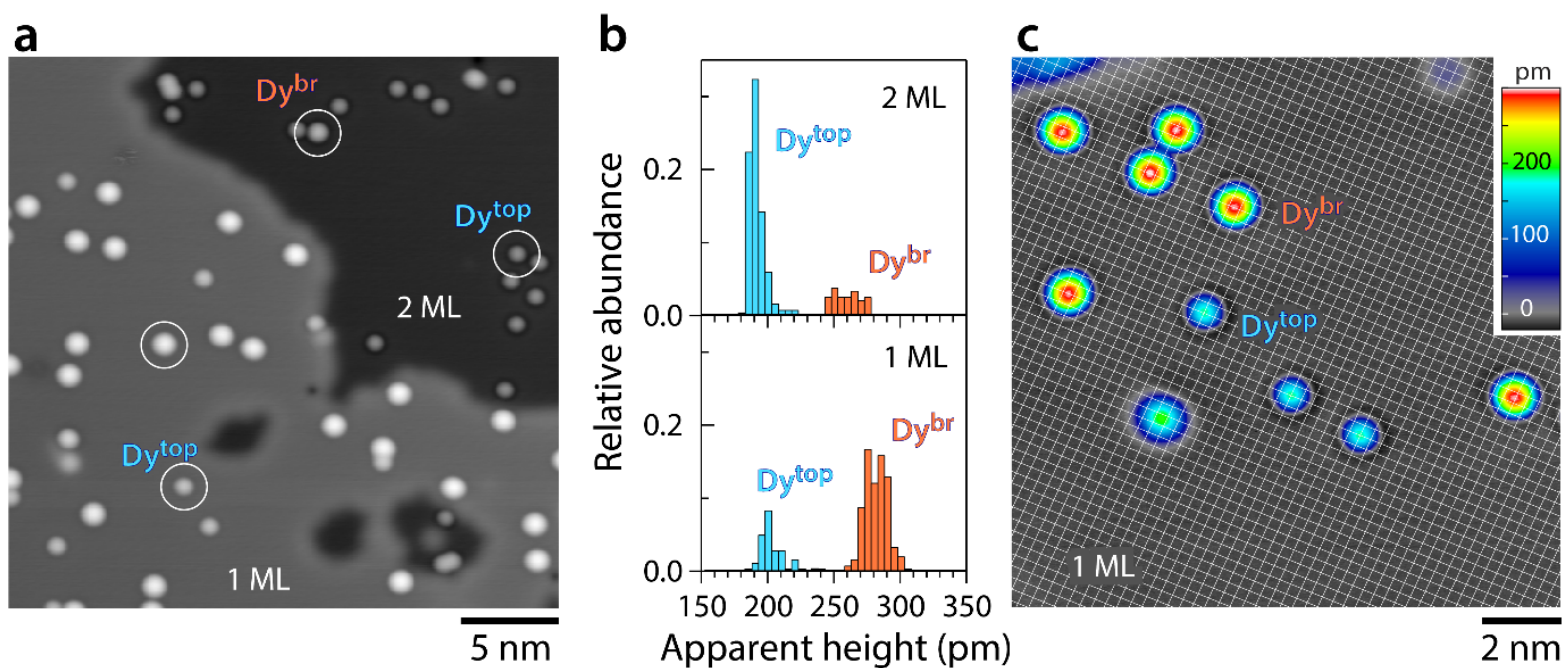

Figure 2. Scanning tunneling microscopy of Dy atoms on $\mathrm{MgO} / \mathrm{Ag}(100)$. (a) After deposition on the $\mathrm{MgO}$ surface kept at around $10 \mathrm{~K}$, two Dy species with different apparent height are observed in STM images. (b) Apparent height distributions of the two Dy species for 1 and 2 ML MgO. (c) By overlapping a grid representative of the $\mathrm{O}$ sub-lattice, corresponding to the crossings of the grid, we identify the two species as Dy atoms adsorbed on O-top (Dy ${ }^{\text {top }}$ ) and on bridge (Dy ${ }^{\text {br }}$, respectively. The grid is obtained from an atomically resolved image of the $\mathrm{MgO}$ surface in a spot without Dy atoms. The species with elongated shape on the bottom-left side of the image is tentatively identified as a Dy dimer $\left(\Theta_{\text {Dy }}=0.007 \mathrm{ML}, \mathrm{V}_{\mathrm{t}}=-100 \mathrm{mV}, \mathrm{I}_{\mathrm{t}}=20 \mathrm{pA}, \mathrm{T}=5 \mathrm{~K}\right)$.

In order to simulate the $\mathrm{XAS} / \mathrm{XMCD}$ spectra and to derive quantitative information on the abundance of the different electronic configurations, we investigate the adsorption sites of Dy atoms using STM for the $\mathrm{MgO}$ thickness range accessible to this technique. On ultra-thin $\mathrm{MgO}$ layers on $\mathrm{Ag}(100)$, Dy atoms appear as protrusions with two distinct apparent heights, whose precise values depend on the $\mathrm{MgO}$ layer thickness (see Figure 2a and 2b). Overlaying the $\mathrm{MgO}$ lattice deduced from atomic-resolution images allows attributing the 2-fold bridge adsorption site to the species with the largest apparent height and a 4-fold symmetric site to the other ones, see 
Figure 2c. Further samples prepared by co-depositing Co and Dy allow ascertaining the 4-fold adsorption site as O-top (see Fig. S1) $3,35,36$. The abundances of the two populations, hereafter being referred to as $\mathrm{Dy}^{\mathrm{br}}$ and Dy ${ }^{\text {top }}$, respectively, vary with the thickness of the MgO layer. On 1 ML MgO, Dy ${ }^{\text {br }}$ atoms $(79 \pm 4 \%)$ are more abundant than $\mathrm{Dy}^{\text {top }}$ ones $(21 \pm 4 \%)$, whereas on $2 \mathrm{ML}$ thick regions the abundances are essentially reversed, with $25 \pm 5 \% \mathrm{Dy}^{\mathrm{br}}$ and $75 \pm 5 \% \mathrm{Dy}^{\text {top }}$. A comparable ratio is also found on $3 \mathrm{ML} \mathrm{MgO}$. This abrupt change of adsorption site abundances between 1 and $2 \mathrm{MgO}$ layers seems dissociated from the smooth variation from $4 \mathrm{f}^{9}$ to $4 \mathrm{f}^{10}$ population occurring over a broader range of $\mathrm{t}_{\mathrm{MgO}}$, suggesting that the change in $4 \mathrm{f}$ occupation has a different origin. Similar variations in the relative population of O-top and bridge were also found for Ho atoms and ascribed to the layer-dependent transient mobility after surface adsorption ${ }^{35,36}$. However, compared to $\mathrm{Ho}$, for the same $\mathrm{MgO}$ thickness Dy atoms show a larger abundance of bridge species ${ }^{35,} 36$ that need to be considered to interpret ensemble-averaged XAS/XMCD measurements.

To quantify the abundance of species with $4 \mathrm{f}^{9}$ and $4 \mathrm{f}^{10}$ configuration, we fit the angle-dependent XAS for each $\mathrm{MgO}$ thickness with simulated spectra from multiplet calculations using the MultiX code ${ }^{37}$, including the adsorption site specific $\mathrm{C}_{4 \mathrm{v}}$ - and $\mathrm{C}_{2 \mathrm{v}}$ - symmetric crystal field (CF) from a point charge model, with the positions and values of the point charges are deduced from DFT Born charges, as discussed in SI. Although the STM measurements are carried out on ultra-thin MgO films, we assume that both adsorption sites are present also on thicker ones with both $4 \mathrm{f}^{9}$ and $4 \mathrm{f}^{10}$ occupations. This procedure provides us with spectra characteristic of each of the four electronic configuration - adsorption site pairs, which we combine to reproduce the experiments (see Fig. S2 and S3). Our fits reproduce very well the angle-dependent XAS and provides the abundance of each species as a function of the thickness of the $\mathrm{MgO}$ film (Table S5), with the overall $4 \mathrm{f}^{9}$ and 
$4 \mathrm{f}^{10}$ fractions shown in Figure 1c. The $4 \mathrm{f}^{9}$ fraction changes smoothly from $84 \%$ for the thinnest $\mathrm{MgO}$ layer to $38 \%$ for the thickest one, with the crossover between the two electronic configurations occurring at around 6-7 $\mathrm{ML}$ of $\mathrm{MgO}$. The smooth trend is a consequence of the roughness of the $\mathrm{MgO}$ films on $\operatorname{Ag}(100)^{38}$.

The ability to tune the electronic configuration by varying the $\mathrm{MgO}$ film thickness gives us the unique opportunity to characterize the magnetic properties of the Dy in $4 \mathrm{f}^{9}$ and $4 \mathrm{f}^{10}$ electronic states while keeping the same crystal field. Indeed, we observe a different magnetic behavior as highlighted by the angular and magnetic field dependence of the XMCD. The difference between normal and grazing incidence XMCD signals is almost negligible at the lowest $\mathrm{MgO}$ thickness where the $4 \mathrm{f}^{9}$ signal dominates, while it becomes more pronounced when the relative weight of the $4 \mathrm{f}^{10}$ configuration is larger, see Fig. $1 \mathrm{~b}$.

Pronounced angular dependence of the XMCD with largest signal in normal incidence is the fingerprint of species with large perpendicular magnetic anisotropy $y^{3,4,11,12,17,18,21,34,39-41}$. The absence of out-of-plane anisotropy for the $4 \mathrm{f}^{9}$ species is in apparent contrast with previous STM studies finding record-high values of uniaxial perpendicular anisotropy for Dy ${ }^{\text {top }}$ on $2 \mathrm{ML}$ $\mathrm{MgO} / \mathrm{Ag}(100)^{8}$, the largest among surface spins. However, due to $4 \mathrm{f}^{9} \mathrm{Dy}{ }^{\mathrm{br}}$ atoms with a larger XMCD along the grazing orientation, the directional contribution of the two $4 \mathrm{f}^{9}$ species cancels out, resulting in a very small angular dependence of the overall $4 \mathrm{f}^{9} \mathrm{XMCD}$. On the other hand, the pronounced angular dependence at the $4 \mathrm{f}^{10}$ peak indicates that both $4 \mathrm{f}^{10} \mathrm{Dy}^{\mathrm{top}}$ and $\mathrm{Dy}{ }^{\mathrm{br}}$ have perpendicular anisotropy, as also predicted by multiplet calculations (see Fig. S2). 


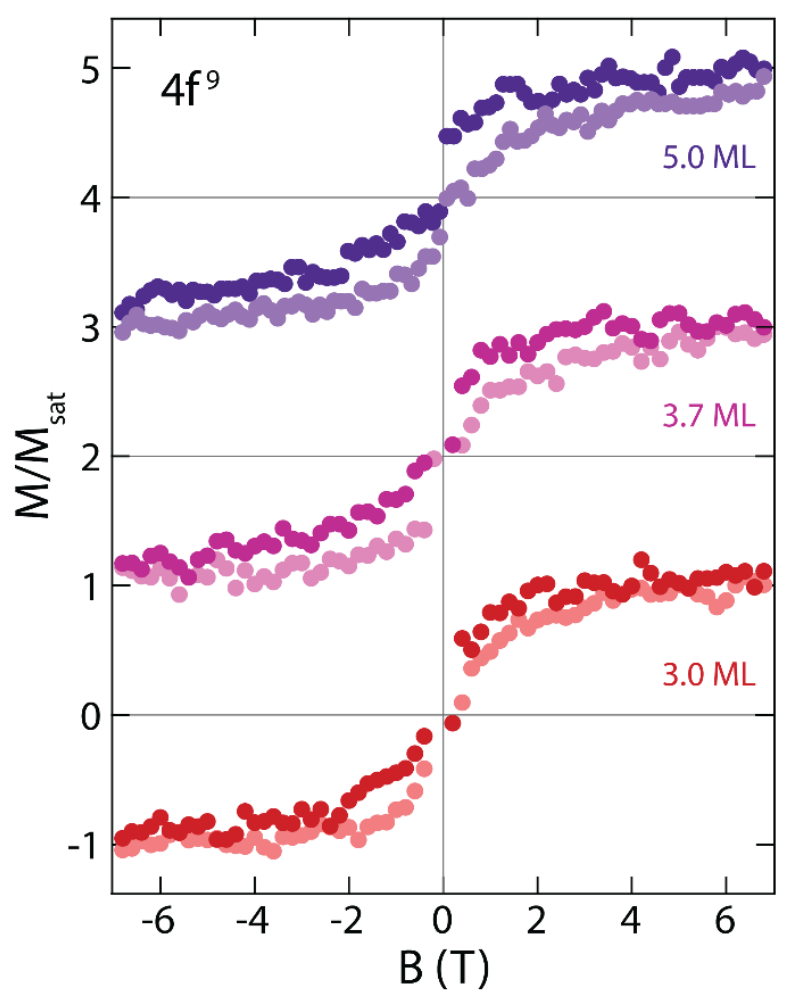

Figure 3. Out-of-plane hysteresis loops of Dy $4 \mathrm{f}^{9}$ species for different $\mathrm{MgO}$ thicknesses. The points represent the out-of-plane XMCD at the Dy $4 \mathrm{f}^{9}$ peak $(1290.5 \mathrm{eV})$ acquired while sweeping the magnetic field at $33.3 \mathrm{mT} / \mathrm{s}\left(\Theta_{\mathrm{Dy}}=0.009 \div 0.014 \mathrm{ML}, \mathrm{T}=2.5 \mathrm{~K}\right)$.

In order to evaluate the magnetic stability of the individual species, we acquire hysteresis loops by measuring the intensity of the XMCD while sweeping the external magnetic field. The energy difference in the XMCD peak related to the two electronic configurations allows resolving the field-dependent magnetization separately for the $4 \mathrm{f}^{9}$ and $4 \mathrm{f}^{10}$ species. Figure 3 shows the out-ofplane magnetization loops of the Dy $4 \mathrm{f}^{9}$ species acquired by measuring the XMCD signal at 1290.5 $\mathrm{eV}$ as a function of the magnetic field sweeping from $+6.8 \mathrm{~T}$ to $-6.8 \mathrm{~T}$ and back to $+6.8 \mathrm{~T}$ with a rate of $33.3 \mathrm{mT} / \mathrm{s}$. In all samples we observe a narrow opening, which extends up to the maximum available field. The area of the loop slightly increases with the thickness of the $\mathrm{MgO}$ film, possibly 
due to a larger decoupling from the hot electrons generated in the Ag substrate by X-ray photon absorption $^{42}$. The presence of magnetic hysteresis indicates that the magnetic relaxation to the ground state is slower than the time resolution of the experiment. According to previous STM results $^{8}$, Dy ${ }^{\text {top }}$ atoms exhibit a long magnetic lifetime up to $5 \mathrm{~T}$, which should result in a wide hysteresis loop. Conversely, $\mathrm{Dy}^{\mathrm{br}}$ atoms, being in a low symmetry $\mathrm{CF}$, are expected to show reduced magnetic lifetime and to exhibit a fully paramagnetic loop. Based on the mixed composition of the ensemble, we interpret the narrow hysteresis as an average behavior between hysteretic Dy ${ }^{\text {top }}$ and paramagnetic Dy ${ }^{\text {br }}$ species.

The out-of-plane hysteresis curve acquired at the XMCD peak of the $4 \mathrm{f}^{10}$ species $(1288.3 \mathrm{eV})$ reveals a very different shape and pronounced anisotropy, see Figures 4a and S4. The loop is wide open up to $3 \mathrm{~T}$, with a strong signature of QTM at zero external field. Similar signature of QTM was also observed for Dy(II) $4 \mathrm{f}^{9} 5 \mathrm{~d}^{1}$ in single ion magnets ${ }^{22}$ and ascribed to the non-Kramers nature of the ion, which is more sensitive to deviations from a perfect axial environment ${ }^{31}$. The low symmetry adsorption geometry of the $4 \mathrm{f}^{10} \mathrm{Dy}{ }^{\text {br }}$ is not expected to enable long magnetic lifetimes, similarly to $4 \mathrm{f}^{9}$ Dy ${ }^{\text {br }}$ discussed above. Hence, we attribute the hysteretic behavior of the $4 \mathrm{f}^{10}$ species to the Dy ${ }^{\text {top }}$ atoms. 

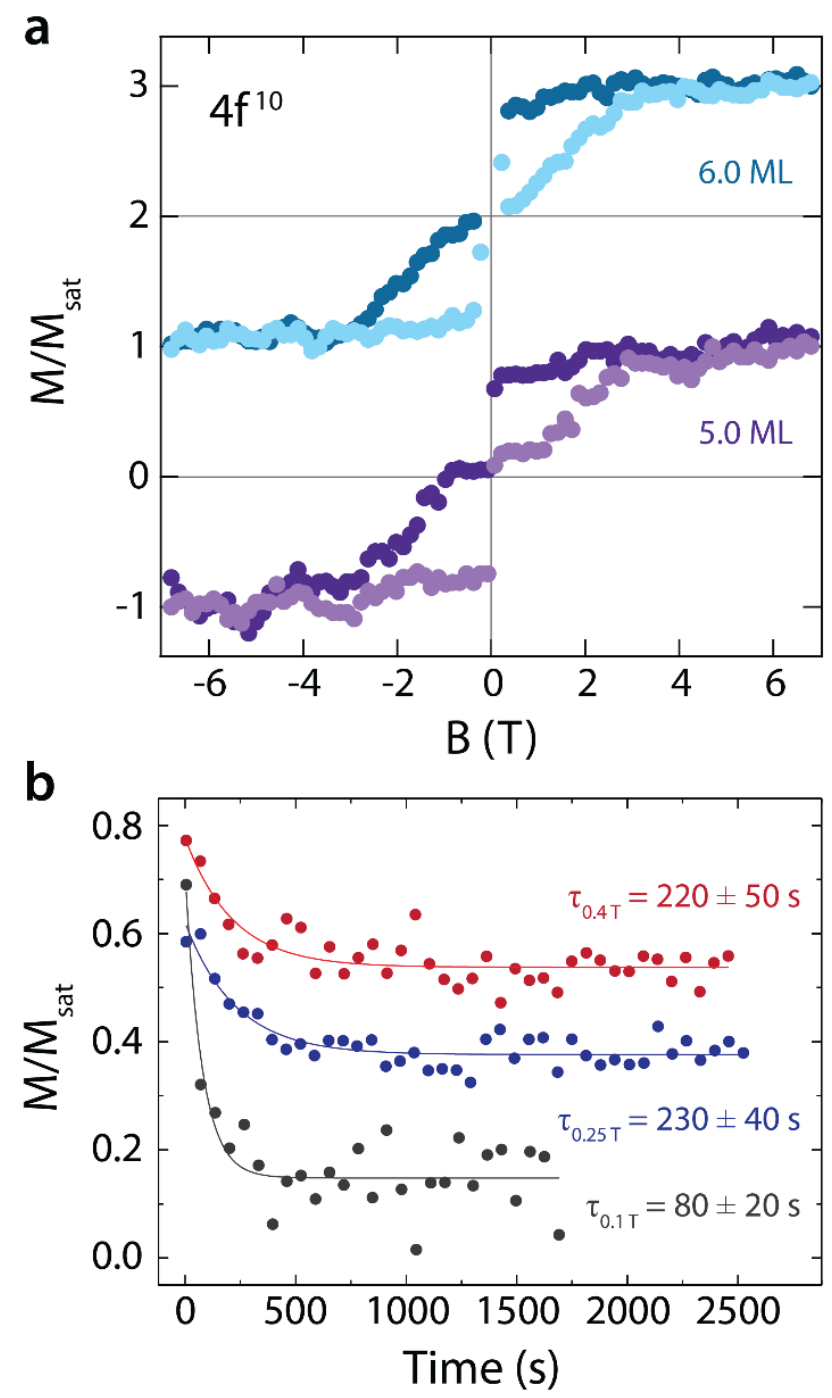

Figure 4. Out-of-plane hysteresis loops and magnetic relaxation of Dy $4 \mathrm{f}^{10}$ species. (a) The points represent the out-of-plane XMCD at the Dy $4 \mathrm{f}^{10}$ peak $(1288.3 \mathrm{eV})$ acquired while sweeping the magnetic field at $33.3 \mathrm{mT} / \mathrm{s}$, for different $\mathrm{MgO}$ thicknesses. (b) Decay of the normal incidence $\mathrm{XMCD}$ signal as a function of time for $\mathrm{B}=0.4 \mathrm{~T}$ (red dots), $\mathrm{B}=0.25 \mathrm{~T}$ (blue dots), and $\mathrm{B}=0.1 \mathrm{~T}$ (black dots) after saturating the ensemble at $3 \mathrm{~T}$ on a sample with $6.0 \mathrm{ML} \mathrm{MgO}$. Lines are exponential fits to the data providing the magnetic lifetime $\tau$ at the related field $\left(\Theta_{\mathrm{Dy}}=0.009 \div\right.$ $0.012 \mathrm{ML}, \mathrm{T}=2.5 \mathrm{~K}$ ). 
To quantify the lifetime of the $4 \mathrm{f}^{10}$ Dy ${ }^{\text {top }}$, we record the time decay of the XMCD signal at constant external field after saturating the magnetization in a field of $3 \mathrm{~T}$ prior to switching the field to the indicated values and starting the acquisition ${ }^{3,4,18,40,42,43}$. Figure $4 \mathrm{~b}$ shows the magnetic relaxation at three different external fields, with the asymptotic value of the XMCD signal indicating the corresponding equilibrium magnetization of the ensemble. The $4 \mathrm{f}^{10}$ species show a magnetic lifetime of about $200 \mathrm{~s}$ at both $0.25 \mathrm{~T}$ and $0.4 \mathrm{~T}$, while lowering the field to $0.1 \mathrm{~T}$ shortens the relaxation time to less than $100 \mathrm{~s}$ due to QTM becoming more effective in this field range, as observed for other non-Kramers single molecule magnets ${ }^{22}$. Magnetic relaxation at lower fields becomes too fast to be detected with our setup. The lifetime of $4 \mathrm{f}^{10}$ Dy atoms on $\mathrm{MgO}$ is a few orders of magnitude shorter than that reported for $4 \mathrm{f}^{9} \mathrm{Dy}^{\text {top }}$ on $2 \mathrm{ML} \mathrm{MgO} / \mathrm{Ag}(100)^{8}$, and for other Ho and Dy single atom magnets with the same $4 \mathrm{f}_{\text {occupation }}{ }^{3,12,43}$, but similar to that of divalent Dy $4 \mathrm{f}^{9} 5 \mathrm{~d}^{1}$ single molecule magnets in polycrystalline form ${ }^{22}$.

In order to unravel the mechanism determining the variation of the Dy $4 \mathrm{f}$ orbital filling with the thickness of the supporting $\mathrm{MgO}$ layer, we perform DFT calculations using pseudopotentials and plane wave bases, as implemented in Quantum Espresso. ${ }^{44-49}$ To correct for the self-interaction of the exchange-correlation functional, we applied a Hubbard $\mathrm{U}$ correction of $9 \mathrm{eV}$ on both the $4 \mathrm{f}$ and $5 \mathrm{~d}$ manifolds. ${ }^{50,51}$ To understand the role of the adsorption site and of the $\operatorname{Ag}(100)$ substrate, we simulate the Dy ${ }^{\text {top }}$ and $\mathrm{Dy}^{\mathrm{br}}$ species on both free-standing $2 \mathrm{ML} \mathrm{MgO}(100)$ and $2 \mathrm{ML}$ $\mathrm{MgO} / \mathrm{Ag}(100)$. When adsorbed on O-top on $\mathrm{MgO}(100)$ (Figure 5a), the Dy atoms show an atomiclike $4 \mathrm{f}^{10}$ occupation, with a slight electron transfer of $0.4 e$ to the $\mathrm{MgO}$, mostly from the outer $6 \mathrm{~s}$ shell. Differently, on $\mathrm{MgO} / \mathrm{Ag}(100)$ the presence of the $\mathrm{Ag}$ layer induces a reorganization of the electronic configuration to $4 f^{9.3}$ with almost one electron transferred from the $4 \mathrm{f}$ orbitals to the $\mathrm{Ag}$ substrate (Figure 5b). The corresponding orbital-projected density of states (PDOS) of the Dy ${ }^{\text {top }}$ 
species reveals a different occupation of the $4 \mathrm{f}$ minority states close to the Fermi level, as highlighted in Figures 5c and 5d. When Dy is adsorbed on free-standing MgO, we observe a peak in the 4f PDOS below the Fermi level, which moves to the unoccupied states on $\mathrm{MgO} / \mathrm{Ag}(100)$. Our calculations reveal that the reorganization of the $4 \mathrm{f}$ charge is driven by two factors: i) more pronounced electron transfer from the Dy to the substrate when the $\operatorname{Ag}(100)$ is included ( $0.4 e$ for $\mathrm{MgO}, 0.7 e$ for $\mathrm{MgO} / \mathrm{Ag}(100))$, ii) shortening of the bond length to the underneath $\mathrm{O}$ from $2.2 \AA$ for $\mathrm{MgO}$ to $2.1 \AA$ for $\mathrm{MgO} / \mathrm{Ag}(100)$, as also indicated in Figs. 5a and $5 \mathrm{~b}$, and Table $\mathrm{S} 7$. Remarkably, both the orbital occupation and bond length of $\mathrm{Dy} / \mathrm{MgO} / \mathrm{Ag}(100)$ are essentially identical to that of $[\mathrm{Dy} / \mathrm{MgO}]^{+}$, where the total charge of the system is artificially reduced by one unit (see SI). Very similar behavior is also observed in the calculations for the bridge site (see SI). This similarity suggests a simplified picture, with the Dy atoms on $\mathrm{MgO} / \mathrm{Ag}(100)$ assuming a singly ionized configuration and those on free-standing $\mathrm{MgO}$ films keeping a close to neutral state. As also observed for pentacene on $\mathrm{MgO} / \mathrm{Ag}(100)$, the gradual neutralization of the charged species with increasing $\mathrm{MgO}$ thickness can be attributed to the increase of electrostatic energy as in an equivalent charged-capacitor scheme ${ }^{52}$, which progressively reduces the energy gain associated to the charging from the electron transfer. Using a plane capacitor model, we infer that an electrostatic energy of about $150 \mathrm{meV}$ per Dy atom is required to overcome the configurational energy difference between $4 \mathrm{f}^{9}$ and $4 \mathrm{f}^{10}$ occupation, a value of comparable magnitude with respect to previous estimations for Dy divalent compounds ${ }^{15}$. 
a

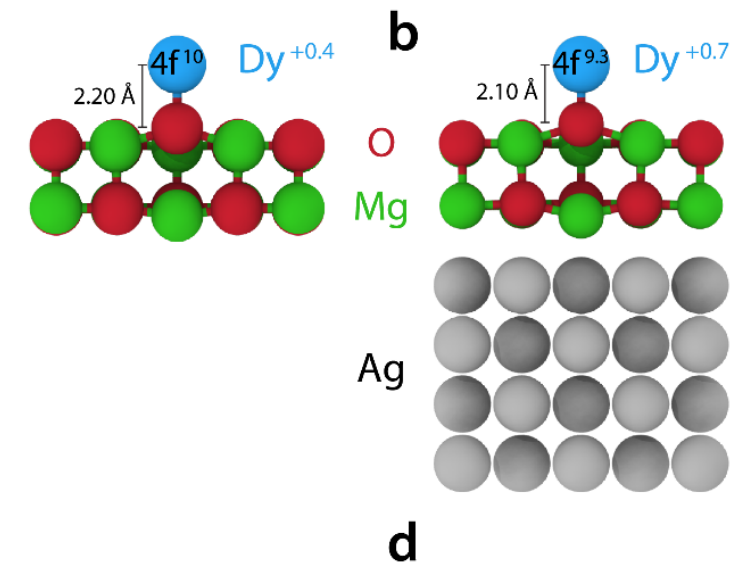

C
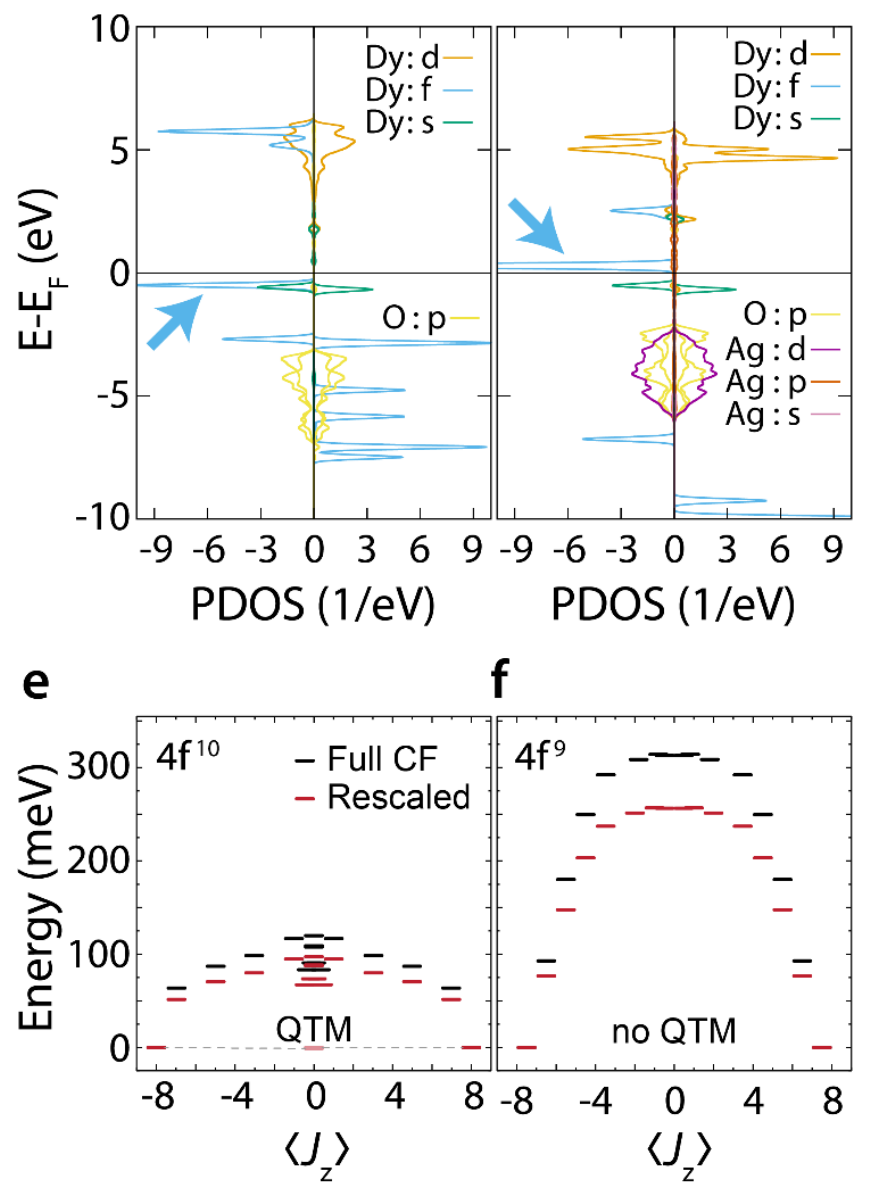

Figure 5. Calculated adsorption geometry, as well as electronic and magnetic configurations of Dy ${ }^{\text {top }}$ atoms. Cross section of the Dy/surface adsorption complex along the crystallographic (010) direction on (a) $2 \mathrm{ML}$ free-standing $\mathrm{MgO}(100)$ and (b) $2 \mathrm{ML} \mathrm{MgO} / \mathrm{Ag}(100)$, showing the charge state, 4f occupation, and Dy-O bond length. PDOS of Dy atoms on (c) $\mathrm{MgO}(100)$, and (d) $\mathrm{MgO} / \mathrm{Ag}(100)$ obtained from DFT. The thick cyan arrows mark the $4 \mathrm{f}$ states that are mostly 
affected by the presence of the $\operatorname{Ag}(100)$, and determines the change of $4 \mathrm{f}$ occupation in Dy atoms. (e) Energy level scheme for the magnetic states of Dy atoms in the $4 \mathrm{f}^{10}$ configuration obtained from multiplet calculations using the structure calculated in (a). Dashed lines show the ground state mixing at zero field, producing two singlet states with no net magnetization (light red lines). (f) Energy level scheme for the magnetic states of the $4 \mathrm{f}^{9}$ configuration using the structure calculated in (b). In both (e) and (f), black lines are the result of the full CF potential, while red lines are obtained rescaling the CF by a factor 0.8 to match results of previous STM experiment ${ }^{8}$.

Changes in bond length and $4 \mathrm{f}$ occupation have a large impact on the adatom magnetic states and anisotropy barrier. For $4 \mathrm{f}^{10}$ Dy ${ }^{\text {top }}$, multiplet calculations indicate a lowest manifold with total angular momentum $\mathrm{J}=8$ and relatively large level splitting of $120 \mathrm{meV}$, see Fig. 5e. For these non-Kramers spins, the non-axial crystal field terms generated by the nearest $\mathrm{Mg}$ neighbors mix the states of the lowest doublet with projected moment $J_{z}= \pm 8$ in a pair of non-degenerate singlets at zero magnetic field, resulting in QTM. Conversely, for the $4 \mathrm{f}^{9} \mathrm{Dy}^{\text {top }}$ atoms we find a lowest manifold with $J=15 / 2$ and giant anisotropy barrier of $314 \mathrm{meV}$, see Fig. $5 f$. For these Kramers spins, the $J_{Z}= \pm 15 / 2$ levels belonging to the lowest doublet cannot be mixed by crystal field terms due to time-reversal symmetry. Therefore, the ground state doublet is protected against QTM at zero magnetic field. The lack of QTM and the giant magnetic anisotropy identify the $4 \mathrm{f}^{9} \mathrm{Dy}^{\text {top }}$ as an extremely stable quantum magnet, in agreement with previous findings ${ }^{8}$. Conversely, Dy ${ }^{\text {br }}$ species show strong mixing of the magnetic states due to the absence of axial symmetry (see Fig. S7), hence lacking the prerequisite for magnetic stability. The apparent contradictory observation of a smaller opening in the magnetization curve of $4 \mathrm{f}^{9} \mathrm{vs} .4 \mathrm{f}^{10}$ species originates from the fact that the $4 \mathrm{f}^{9}$ Dy ${ }^{\text {top }}$ lifetime is so long and weakly affected by the magnetic field that the spin dynamics 
is basically negligible in the time lapse of a hysteresis. Even in the presence of secondary electrons that can accelerate the dynamics of the spin state ${ }^{3,40,42}$, the $4 \mathrm{f}^{9} \mathrm{Dy}^{\text {top }}$ population does not seem to attain a large unbalance between the two bistable spin orientations, hence the contribution to the $4 \mathrm{f}^{9}$ magnetization remains small. Consequently, the data on thin $\mathrm{MgO}$ films are dominated by the S-shaped curve of the paramagnetic $4 \mathrm{f}^{9}$ Dy ${ }^{\mathrm{br}}$ atoms.

We finally note that the anisotropy barrier of $4 \mathrm{f}^{9} \mathrm{Dy} \mathrm{y}^{\text {top }}$ predicted by our multiplet calculations is $25 \%$ larger than the value of $258 \mathrm{meV}\left(2081 \mathrm{~cm}^{-1}, 2994 \mathrm{~K}\right)$ inferred from STM expriments ${ }^{8}$, possibly due to the well-known inaccuracy of point charge models based on physical charge distribution $^{53}$. By rescaling the $\mathrm{CF}$ charges of all species to match the previously reported barrier for $4 \mathrm{f}^{9}$ Dy ${ }^{\text {top }}$, we obtain a reduced anisotropy barrier of $97 \mathrm{meV}\left(782 \mathrm{~cm}^{-1}, 1126 \mathrm{~K}\right)$ for the $4 \mathrm{f}^{10}$ Dy ${ }^{\text {top }}$ (red lines in Figs. 5e and 5f). For the latter species, the rescaled barrier height results to be smaller than the one estimated for $4 \mathrm{f}^{10} \mathrm{Ho}$ atoms on $\mathrm{MgO}^{24,26}$, in spite of their identical $4 \mathrm{f}$ filling. The reason can be traced to the more extended $\mathrm{Dy}^{\text {top }}-\mathrm{O}$ bond when Dy is in its neutral state, which reduces the uniaxial anisotropy component and hence the robustness of the magnetic states against QTM and spin-phonon scattering ${ }^{31}$.

Recent calculations for DyO linear complexes find anisotropy barriers of $4 \mathrm{f}^{9}$ and $4 \mathrm{f}^{10}$ electronic configurations comparable with our results ${ }^{31,32}$. The lower magnetic stability at zero field observed for the $4 \mathrm{f}^{10}$ Dy ${ }^{\text {top }}$ with respect to the previously reported long lifetime of $4 \mathrm{f}^{10}$ $\mathrm{Dy} / \mathrm{gr} / \mathrm{Ir}(111)$ is a consequence of the crystal field symmetry ${ }^{4,12}$. The adsorption on the graphene hollow site produces a $\mathrm{C}_{6 \mathrm{v}}$ symmetric $\mathrm{CF}$ resulting in a degenerate ground state doublet characterized by $\mathrm{J}_{Z}=7$, and thus protected from QTM. On the contrary, in the present case, the $\mathrm{C}_{4 \mathrm{v}}$ symmetric CF produces a ground split-doublet exhibiting QTM in vanishing magnetic field. 
Electron transfer from metal supports through ultra-thin $\mathrm{MgO}$ films has been observed for molecular adsorbates ${ }^{52,54}$ and Au atoms ${ }^{36,54,55}$. The lower ionization potential of lanthanides offers the possibility to realize the opposite transfer mechanism, i.e., from the adatom to the metal substrate. As shown in a previous study, such a mechanism occurs for Gd and Ho atoms, leaving the adsorbate in a singly ionized configuration ${ }^{24}$. Our findings reveal a very similar behavior for Dy: the charge transfer removes one electron from the $4 \mathrm{f}$ orbitals leading to a $4 \mathrm{f}^{9}$ occupation. In this configuration, adsorption on O-top gives rise to the highest magnetic anisotropy barrier observed for surface spins, and absence of QTM. Increasing the thickness of the oxide layer reduces the energy gain associated to this process ${ }^{52}$, inducing the Dy atoms to gradually recover a neutral atomic-like configuration. Although characterized by a reduced perpendicular anisotropy compared with the $4 \mathrm{f}^{9}$ Dy ${ }^{\text {top }}$ case, also the $4 \mathrm{f}^{10}$ Dy ${ }^{\text {top }}$ species exhibit long magnetic lifetimes and open hysteresis. Our results prove the charge transfer to be a controllable mechanism to tune the magnetic behavior in surface-adsorbed spins.

\section{ASSOCIATED CONTENT}

\section{Supporting Information.}

This material is available free of charge via the internet at http://pubs.acs.org.

"Donati_SI_submitted.pdf(PDF)" : additional information on the sample preparation, STM and XAS/XMCD measurements, and the related analysis, additional details on the DFT and multiplet calculations. 


\section{AUTHOR INFORMATION}

\section{Corresponding Authors}

*donati.fabio@qns.science, stefano.rusponi@epfl.ch

\section{Author Contributions}

F.D., M.P., A.S., C.Wä., R.B., S.L.A., L.P., C.N., J.D., A.B., and S.R. performed the XAS/XMCD synchrotron experiments under the supervision of P.G. and H.B. The analysis of the XAS/XMCD data using multiplet calculations have been performed by F.D. with the help of S.L.A. STM experiments have been performed and analyzed by M.P., J.-G.de G., and E.F. DFT calculations have been performed by C.Wo. The manuscript was written by F.D., M.P., C.Wo., and S.R. and finalized through contributions of all authors. All authors have given approval to the final version of the manuscript.

\section{Funding Sources}

Institute for Basic Science (IBS-R027-D1), SNF Ambizione (PZ00P2_142474).

\section{Notes}

The authors declare no competing financial interest.

\section{ACKNOWLEDGMENT}

F.D, C.Wo., A.S., and S.L.A. acknowledge support from the Institute for Basic Science under Grant IBS-R027-D1. C.Wä. and J.D. acknowledge funding from SNF Ambizione through the Grant no. PZ00P2_142474. 


\section{ABBREVIATIONS}

STM, scanning tunneling microscopy; XAS, X-ray absorption spectroscopy; XMCD, X-ray circular magnetic dichroism; $\mathrm{t}_{\mathrm{MgO}}$, average thickness of the $\mathrm{MgO}$ layer; $\Theta_{\mathrm{Dy}}$, Dy coverage; Dy ${ }^{\text {top }}$, Dy atoms adsorbed on O-top site of $\mathrm{MgO}$; $\mathrm{Dy}^{\text {br }}$, Dy atoms adsorbed on bridge site of $\mathrm{MgO} ; \mathrm{CF}$, crystal field; DFT, density functional theory; PDOS, projected density of states; QTM, quantum tunneling of the magnetization.

\section{REFERENCES}

1. Goodwin, C. A. P.; Ortu, F.; Reta, D.; Chilton, N. F.; Mills, D. P., Molecular magnetic hysteresis at 60 kelvin in dysprosocenium. Nature 2017, 548 (7668), 439-442.

2. Guo, F. S.; Day, B. M.; Chen, Y. C.; Tong, M. L.; Mansikkamaki, A.; Layfield, R. A., Magnetic hysteresis up to 80 kelvin in a dysprosium metallocene single-molecule magnet. Science 2018, 362 (6421), 1400-1403.

3. $\quad$ Donati, F.; Rusponi, S.; Stepanow, S.; Wäckerlin, C.; Singha, A.; Persichetti, L.; Baltic, R.; Diller, K.; Patthey, F.; Fernandes, E.; Dreiser, J.; Sljivancanin, Z.; Kummer, K.; Nistor, C.; Gambardella, P.; Brune, H., Magnetic remanence in single atoms. Science 2016, 352 (6283), 318-21.

4. $\quad$ Baltic, R.; Pivetta, M.; Donati, F.; Wäckerlin, C.; Singha, A.; Dreiser, J.; Rusponi, S.; Brune, H., Superlattice of Single Atom Magnets on Graphene. Nano Lett. 2016, 16 (12), 76107615.

5. Ishikawa, N.; Sugita, M.; Ishikawa, T.; Koshihara, S.; Kaizu, Y., Lanthanide doubledecker complexes functioning as magnets at the single-molecular level. J. Am. Chem. Soc. 2003, 125 (29), 8694-8695.

6. $\quad$ Dreiser, J., Molecular lanthanide single-ion magnets: from bulk to submonolayers. $J$ Phys Condens Matter 2015, 27 (18), 183203.

7. Donati, F., Magnetic Relaxation Mechanisms in Ho Single Atom Magnets. J. Magn. 2020, 25 (4), 441-452.

8. $\quad$ Singha, A.; Willke, P.; Bilgeri, T.; Zhang, X.; Brune, H.; Donati, F.; Heinrich, A. J.; Choi, T., Engineering atomic-scale magnetic fields by dysprosium single atom magnets. Nat. Commun. 2021, 12 (1), 4179.

9. $\quad$ Thiele, S.; Balestro, F.; Ballou, R.; Klyatskaya, S.; Ruben, M.; Wernsdorfer, W., Electrically driven nuclear spin resonance in single-molecule magnets. Science 2014, 344 (6188), $1135-8$.

10. Pedersen, K. S.; Ariciu, A. M.; McAdams, S.; Weihe, H.; Bendix, J.; Tuna, F.; Piligkos, S., Toward Molecular 4f Single-Ion Magnet Qubits. J. Am. Chem. Soc. 2016, 138 (18), $5801-4$.

11. Singha, A.; Baltic, R.; Donati, F.; Wäckerlin, C.; Dreiser, J.; Persichetti, L.; Stepanow, S.; Gambardella, P.; Rusponi, S.; Brune, H., 4f-occupancy and magnetism of rareearth atoms adsorbed on metal substrates. Phys. Rev. B 2017, 96 (22). 
12. Baltic, R.; Donati, F.; Singha, A.; Wäckerlin, C.; Dreiser, J.; Delley, B.; Pivetta, M.;

Rusponi, S.; Brune, H., Magnetic properties of single rare-earth atoms on graphene/Ir(111).

Phys. Rev. B 2018, 98 (2).

13. Jaroschik, F.; Nief, F.; Le Goff, X.-F.; Ricard, L., Isolation of Stable

Organodysprosium(II) Complexes by Chemical Reduction of Dysprosium(III) Precursors.

Organometallics 2007, 26 (5), 1123-1125.

14. Moutet, J.; Schleinitz, J.; La Droitte, L.; Tricoire, M.; Pointillart, F.; Gendron, F.; Simler, T.; Clavaguéra, C.; Le Guennic, B.; Cador, O.; Nocton, G., Bis-Cyclooctatetraenyl Thulium(II): Highly Reducing Lanthanide Sandwich Single-Molecule Magnets. Angew. Chem. Int. Ed. Engl. 2021.

15. Fieser, M. E.; MacDonald, M. R.; Krull, B. T.; Bates, J. E.; Ziller, J. W.; Furche, F.; Evans, W. J., Structural, Spectroscopic, and Theoretical Comparison of Traditional vs Recently Discovered Ln2+ Ions in the [K(2.2.2-cryptand)][(C5H4SiMe3)3Ln] Complexes: The Variable Nature of Dy2+ and Nd2+. J. Am. Chem. Soc. 2015, 137 (1), 369-382.

16. Nistor, C.; Mugarza, A.; Stepanow, S.; Gambardella, P.; Kummer, K.; Diez-Ferrer, J. L.; Coffey, D.; de la Fuente, C.; Ciria, M.; Arnaudas, J. I., Structure and magnetism of Tm atoms and monolayers on $\mathrm{W}(110)$. Phys. Rev. B 2014, 90 (6).

17. Donati, F.; Singha, A.; Stepanow, S.; Wäckerlin, C.; Dreiser, J.; Gambardella, P.; Rusponi, S.; Brune, H., Magnetism of Ho and Er atoms on close-packed metal surfaces. Phys. Rev. Lett. 2014, 113 (23), 237201.

18. Singha, A.; Donati, F.; Wäckerlin, C.; Baltic, R.; Dreiser, J.; Pivetta, M.; Rusponi, S.; Brune, H., Magnetic Hysteresis in Er Trimers on Cu(111). Nano Lett. 2016, 16 (6), 3475-81.

19. Guo, F. S.; Day, B. M.; Chen, Y. C.; Tong, M. L.; Mansikkamaki, A.; Layfield, R. A., A Dysprosium Metallocene Single-Molecule Magnet Functioning at the Axial Limit. Angew. Chem. Int. Ed. Engl. 2017, 56 (38), 11445-11449.

20. Ishikawa, N.; Sugita, M.; Okubo, T.; Tanaka, N.; Iino, T.; Kaizu, Y., Determination of Ligand-Field Parameters and f-Electronic Structures of Double-Decker Bis(phthalocyaninato)lanthanide Complexes. lnorg. Chem. 2003, 42 (7), 2440-2446.

21. Westerstrom, R.; Dreiser, J.; Piamonteze, C.; Muntwiler, M.; Weyeneth, S.; Brune, H.; Rusponi, S.; Nolting, F.; Popov, A.; Yang, S.; Dunsch, L.; Greber, T., An endohedral singlemolecule magnet with long relaxation times: DySc2N@C80. J. Am. Chem. Soc. 2012, 134 (24), 9840-3.

22. Gould, C. A.; McClain, K. R.; Yu, J. M.; Groshens, T. J.; Furche, F.; Harvey, B. G.; Long, J. R., Synthesis and Magnetism of Neutral, Linear Metallocene Complexes of Terbium(II) and Dysprosium(II). J. Am. Chem. Soc. 2019, 141 (33), 12967-12973.

23. MacDonald, M. R.; Bates, J. E.; Fieser, M. E.; Ziller, J. W.; Furche, F.; Evans, W. J., Expanding Rare-Earth Oxidation State Chemistry to Molecular Complexes of Holmium(II) and Erbium(II). J. Am. Chem. Soc. 2012, 134 (20), 8420-8423.

24. Singha, A.; Sostina, D.; Wolf, C.; Safa; Krylov, D.; Colazzo, L.; Gargiani, P.; Agrestini, S.; Noh, W.-S.; Park, J.-H.; Pivetta, M.; Rusponi, S.; Brune, H.; Andreas; Barla, A.; Donati, F. Orbital-resolved single atom magnetism measured with X-ray absorption spectroscopy arXiv pre-print server [Online], 2020. https://arxiv.org/abs/2012.10972 (accessed 2020-12-20).

25. MacDonald, M. R.; Bates, J. E.; Ziller, J. W.; Furche, F.; Evans, W. J., Completing the series of +2 ions for the lanthanide elements: synthesis of molecular complexes of Pr2+, Gd2+, Tb2+, and Lu2+. J. Am. Chem. Soc. 2013, 135 (26), 9857-68. 
26. Natterer, F. D.; Donati, F.; Patthey, F.; Brune, H., Thermal and Magnetic-Field Stability of Holmium Single-Atom Magnets. Phys. Rev. Lett. 2018, 121 (2), 027201.

27. Pivetta, M.; Patthey, F.; Di Marco, I.; Subramonian, A.; Eriksson, O.; Rusponi, S.; Brune, H., Measuring the Intra-Atomic Exchange Energy in Rare-Earth Adatoms. Phys. Rev. X 2020, 10 (3), 031054.

28. Natterer, F. D.; Yang, K.; Paul, W.; Willke, P.; Choi, T.; Greber, T.; Heinrich, A. J.; Lutz, C. P., Reading and writing single-atom magnets. Nature 2017, 543 (7644), 226-228.

29. Meihaus, K. R.; Fieser, M. E.; Corbey, J. F.; Evans, W. J.; Long, J. R., Record High Single-Ion Magnetic Moments Through $4 \mathrm{f}^{\mathrm{n}} 5 \mathrm{~d}^{1}$ Electron Configurations in the Divalent Lanthanide Complexes $\left[\left(\mathrm{C}_{5} \mathrm{H}_{4} \mathrm{SiMe}_{3}\right)_{3} \mathrm{Ln}\right]^{-} . J$. Am. Chem. Soc. 2015, 137 (31), 9855-60.

30. Forrester, P. R.; Patthey, F.; Fernandes, E.; Sblendorio, D. P.; Brune, H.; Natterer, F. D., Quantum state manipulation of single atom magnets using the hyperfine interaction. Phys. Rev. B 2019, 100 (18).

31. Ungur, L.; Chibotaru, L. F., Strategies toward High-Temperature Lanthanide-Based Single-Molecule Magnets. Inorg Chem 2016, 55 (20), 10043-10056.

32. Zhang, W.; Muhtadi, A.; Iwahara, N.; Ungur, L.; Chibotaru, L. F., Magnetic Anisotropy in Divalent Lanthanide Compounds. Angew. Chem. Int. Ed. Engl. 2020, 59 (31), 12720-12724.

33. Piamonteze, C.; Flechsig, U.; Rusponi, S.; Dreiser, J.; Heidler, J.; Schmidt, M.; Wetter, R.; Calvi, M.; Schmidt, T.; Pruchova, H.; Krempasky, J.; Quitmann, C.; Brune, H.; Nolting, F., X-Treme beamline at SLS: X-ray magnetic circular and linear dichroism at high field and low temperature. J. Synchrotron Radiat. 2012, 19 (Pt 5), 661-74.

34. Westerstrom, R.; Uldry, A. C.; Stania, R.; Dreiser, J.; Piamonteze, C.; Muntwiler, M.; Matsui, F.; Rusponi, S.; Brune, H.; Yang, S.; Popov, A.; Buchner, B.; Delley, B.; Greber, T., Surface aligned magnetic moments and hysteresis of an endohedral single-molecule magnet on a metal. Phys. Rev. Lett. 2015, 114 (8), 087201.

35. Fernandes, E.; Donati, F.; Patthey, F.; Stavrić, S.; Šljivančanin, Ž.; Brune, H., Adsorption sites of individual metal atoms on ultrathin $\mathrm{MgO}(100)$ films. Phys. Rev. B 2017, 96 (4).

36. Fernandes, E. Adsorption Sites of Metal Atoms on MgO Thin Films and Rotational Quantum State Spectroscopy of Physisorbed $\mathrm{H}_{2}$. EPFL thesis N ${ }^{\circ} 8144$, Lausanne, 2017.

37. Uldry, A.; Vernay, F.; Delley, B., Systematic computation of crystal-field multiplets for x-ray core spectroscopies. Phys. Rev. B 2012, 85 (12), 125133.

38. Baumann, S.; Rau, I. G.; Loth, S.; Lutz, C. P.; Heinrich, A. J., Measuring the ThreeDimensional Structure of Ultrathin Insulating Films at the Atomic Scale. Acs Nano 2014, 8 (2), 1739-1744.

39. Studniarek, M.; Wäckerlin, C.; Singha, A.; Baltic, R.; Diller, K.; Donati, F.; Rusponi, S.; Brune, H.; Lan, Y.; Klyatskaya, S.; Ruben, M.; Seitsonen, A. P.; Dreiser, J., Understanding the Superior Stability of Single-Molecule Magnets on an Oxide Film. Adv. Sci. 2019, 6 (22), 1901736.

40. Wäckerlin, C.; Donati, F.; Singha, A.; Baltic, R.; Rusponi, S.; Diller, K.; Patthey, F.; Pivetta, M.; Lan, Y.; Klyatskaya, S.; Ruben, M.; Brune, H.; Dreiser, J., Giant Hysteresis of Single-Molecule Magnets Adsorbed on a Nonmagnetic Insulator. Adv. Mater. 2016, 28 (26), 5195-9. 
41. Gambardella, P.; Rusponi, S.; Veronese, M.; Dhesi, S. S.; Grazioli, C.; Dallmeyer, A.; Cabria, I.; Zeller, R.; Dederichs, P. H.; Kern, K.; Carbone, C.; Brune, H., Giant Magnetic Anisotropy of Single Cobalt Atoms and Nanoparticles. Science 2003, 300 (5622), 1130-1133. 42. Dreiser, J.; Westerström, R.; Piamonteze, C.; Nolting, F.; Rusponi, S.; Brune, H.; Yang, S.; Popov, A.; Dunsch, L.; Greber, T., X-ray induced demagnetization of single-molecule magnets. Applied Physics Letters 2014, 105 (3).

43. Donati, F.; Rusponi, S.; Stepanow, S.; Persichetti, L.; Singha, A.; Juraschek, D. M.; Wäckerlin, C.; Baltic, R.; Pivetta, M.; Diller, K.; Nistor, C.; Dreiser, J.; Kummer, K.; VelezFort, E.; Spaldin, N. A.; Brune, H.; Gambardella, P., Unconventional Spin Relaxation Involving Localized Vibrational Modes in Ho Single-Atom Magnets. Phys. Rev. Lett. 2020, 124 (7), 077204.

44. Giannozzi, P.; Baroni, S.; Bonini, N.; Calandra, M.; Car, R.; Cavazzoni, C.; Ceresoli, D.; Chiarotti, G. L.; Cococcioni, M.; Dabo, I.; Dal Corso, A.; de Gironcoli, S.; Fabris, S.; Fratesi, G.; Gebauer, R.; Gerstmann, U.; Gougoussis, C.; Kokalj, A.; Lazzeri, M.; MartinSamos, L.; Marzari, N.; Mauri, F.; Mazzarello, R.; Paolini, S.; Pasquarello, A.; Paulatto, L.; Sbraccia, C.; Scandolo, S.; Sclauzero, G.; Seitsonen, A. P.; Smogunov, A.; Umari, P.;

Wentzcovitch, R. M., QUANTUM ESPRESSO: a modular and open-source software project for quantum simulations of materials. J. Phys.: Condens. Matter 2009, 21 (39), 395502.

45. Giannozzi, P.; Andreussi, O.; Brumme, T.; Bunau, O.; Buongiorno Nardelli, M.; Calandra, M.; Car, R.; Cavazzoni, C.; Ceresoli, D.; Cococcioni, M.; Colonna, N.; Carnimeo, I.; Dal Corso, A.; de Gironcoli, S.; Delugas, P.; DiStasio, R. A.; Ferretti, A.; Floris, A.; Fratesi, G.; Fugallo, G.; Gebauer, R.; Gerstmann, U.; Giustino, F.; Gorni, T.; Jia, J.; Kawamura, M.; Ko, H. Y.; Kokalj, A.; Küçükbenli, E.; Lazzeri, M.; Marsili, M.; Marzari, N.; Mauri, F.; Nguyen, N. L.; Nguyen, H. V.; Otero-de-la-Roza, A.; Paulatto, L.; Poncé, S.; Rocca, D.; Sabatini, R.; Santra, B.; Schlipf, M.; Seitsonen, A. P.; Smogunov, A.; Timrov, I.; Thonhauser, T.; Umari, P.; Vast, N.; Wu, X.; Baroni, S., Advanced capabilities for materials modelling with Quantum ESPRESSO. J. Phys.: Condens. Matter 2017, 29 (46), 465901.

46. Dal Corso, A., Pseudopotentials periodic table: From H to Pu. Comput. Mater. Sci. 2014, 95, 337-350.

47. Prandini, G.; Marrazzo, A.; Castelli, I. E.; Mounet, N.; Marzari, N., Precision and efficiency in solid-state pseudopotential calculations. Npj Comput. Mater. 2018, 4 (1), 72.

48. Blöchl, P. E., Projector augmented-wave method. Phys. Rev. B 1994, 50 (24), $17953-$ 17979.

49. Perdew, J. P.; Burke, K.; Ernzerhof, M., Generalized Gradient Approximation Made Simple. Phys. Rev. Lett. 1996, 77 (18), 3865-3868.

50. Leiria Campo Jr, V.; Cococcioni, M., Extended DFT +U+Vmethod with on-site and inter-site electronic interactions. J. Phys.: Condens. Matter 2010, 22 (5), 055602.

51. Topsakal, M.; Wentzcovitch, R. M., Accurate projected augmented wave (PAW) datasets for rare-earth elements (RE = La-Lu). Comput. Mater. Sci. 2014, 95, 263-270.

52. Hurdax, P.; Hollerer, M.; Puschnig, P.; Lüftner, D.; Egger, L.; Ramsey, M. G.; Sterrer, M., Controlling the Charge Transfer across Thin Dielectric Interlayers. Adv. Mater. Interfaces 2020, 7 (14), 2000592.

53. Goeller-Walrand, C.; Binnemans, K., Rationalization of Crystal-Field Parametrization. In Handbook on the Physics and Chemistry of Rare Earths, Gschneidner, K. A. J.; Eyring, L., Eds. Elsevier: Amsterdam, 1996; Vol. 23, pp 121-283. 
54. Pacchioni, G.; Freund, H., Electron Transfer at Oxide Surfaces. The MgO Paradigm: from Defects to Ultrathin Films. Chem. Rev. 2013, 113 (6), 4035-4072.

55. Sterrer, M.; Risse, T.; Martinez Pozzoni, U.; Giordano, L.; Heyde, M.; Rust, H. P.; Pacchioni, G.; Freund, H. J., Control of the charge state of metal atoms on thin MgO films. Phys. Rev. Lett. 2007, 98 (9), 096107. 